\title{
Cardiovascular Disease Risk Factors Assessment of Women Attending a Religious Program in Ado Ekiti, Nigeria
}

\author{
David Daisi Ajayi ${ }^{1}$, Samson Ayo Deji ${ }^{2,}$, , Olusola Olugbenga Odu ${ }^{2}$, Samuel Ayokunle Dada ${ }^{3}$, \\ Eyitope Oluseyi Amu", Oluwadare Marcus² \\ ${ }^{1}$ Department of Chemical Pathology, College of Medicine, Ekiti State University, Ado Ekiti, Nigeria \\ ${ }^{2}$ Department of Community Medicine, Faculty of Clinical Sciences College of Medicine, Ekiti State University, Ado Ekiti, Nigeria \\ ${ }^{3}$ Department of Medicine, College of Medicine, Ekiti State University, Ado Ekiti, Nigeria
}

Email address:

Samdeji3@hotmail.com (S. A. Deji)

${ }^{*}$ Corresponding author

\section{To cite this article:}

David Daisi Ajayi, Samson Ayo Deji, Olusola Olugbenga Odu, Samuel Ayokunle Dada, Eyitope Oluseyi Amu, Oluwadare Marcus. Cardiovascular Disease Risk Factors Assessment of Women Attending a Religious Program in Ado Ekiti, Nigeria. American Journal of Biomedical and Life Sciences. Vol. 7, No. 2, 2019, pp. 36-41. doi: 10.11648/j.ajbls.20190702.12

Received: March 1, 2019; Accepted: April 12, 2019; Published: May 20, 2019

\begin{abstract}
The burden of cardiovascular diseases in developing countries is alarming and needs urgent attention. The study assessed the prevalence of Cardio Vascular Disease risk factor among women in Ekiti State, Nigeria. The study design was a descriptive cross sectional survey conducted in Ado - Ekiti, Nigeria. Participants recruited for the study through simple random sampling were 426 women who were in a religious outreach program. Interviewer administered semi - structured questionnaires were used to collect information on respondents socio-demographic characteristics, past medical history, nutritional status (using dietary recall), and behaviors related to lifestyle. A general physical examination was done and anthropometric measurements taken from each respondent. The examinations collected data on, blood pressure, weight and height. Blood specimen $(5 \mathrm{ml}$ whole blood) was collected from each respondent for laboratory tests such as random blood sugar (RBS) and serum cholesterol levels. Data from the clinical examinations and laboratory tests were then used to categorize respondents as hypertensive, diabetic, obese and hyperlipidemic. Data was analyzed using SPSS version 20 and level of significance was set at $\mathrm{p}$ values $<0.5$. A total of 426 respondents participated in the survey of which $64.8 \%$ were between $40-59$ years with a mean age of $51.7 \pm 11.9$ years. A high proportion (81.7\%) of respondents had formal education. Most of the respondents (95\%) claimed to be employed. About $51.4 \%$ of the respondents reported history of substance use. The most commonly consumed by respondents were "bitter kola" (31.7\%) and "kolanut" $(9.9 \%)$. About $5 \%$ of respondents either smoked or took substances containing nicotine e.g. "snuff ". Nearly a quarter, $23.2 \%$ of respondents claimed that they have ever used herbal (traditional) medicine (23.2\%) to take care of health issues as the need arises. About $9.2 \%$ of respondents claimed that they occasionally consumed alcoholic drinks. About $49.5 \%$ of the respondents were found to have poor medical history. While majority (73.9\%) of the respondents had normal blood pressure (BP), $12.0 \%$ and $14 \%$ were either prehypertensive or hypertensive. Majority of the respondents, $61.2 \%$, were reported obese with a BMI exceeding 25 . About $63.4 \%$ of respondents had high serum cholesterol while $2.1 \%$ reported smoking habit. There were significant cardiovascular risk factors found among women studied.
\end{abstract}

Keywords: Risk Factors, Cardiovascular, Women, Assessment

\section{Introduction}

There is a rising epidemic of non-communicable diseases (NCDs) in sub-Saharan Africa (SSA) that includes cardiovascular diseases (CVDs), cancers and metabolic diseases such as diabetes and obesity which are far from being diseases exclusively of the wealthy and developed countries alone. The NCDs are already and will continue to be a significant burden on low income countries in the region. [1] In SSA, the epidemiological transition from 
predominantly communicable diseases to NCDs is progressing at a rapid rate and by 2030 will cause $46 \%$ of mortalities. [2].

Current statistics show that CVDs e.g. coronary heart diseases (CHD), myocardial infarctions (MI) and cerebrovascular diseases and accidents (e.g. stroke), - are the leading causes of death in all regions of the world including SSA. [3] However in the region it is projected that between 1990 and 2020 , CHD will increase by $120 \%$ for women and $137 \%$ for men. [4] Recent population studies demonstrated an increasing burden of CVD and related risk factors in sub Saharan Africa. Although HIV/AIDs is still the leading cause of death SSA, CVD is the second overall and first among those over the age of thirty years with hypertension emerging as a major health concern and CVA (stroke) is the dominant form of CVD. [5].

Cardiovascular diseases are rare among people under twenty years of age but commoner with people above forty years of age. While age in itself is important as a causal factor among those with genetic predisposition, the relevant exposure to accumulation of risk factors are responsible for its prevalence in many parts of the world. [6] Additional drivers of the epidemic include changes in life style associated with economic development, reduction in physical activity, smoking and excessive alcohol intake. [7] The impact of the increasing epidemic of CVDs in SSA is both social and economic. The burden of morbidities and mortalities associated with CVD in SSA is more in the younger age group, having a negative effect on the economy and productivity in the region. In high income countries the impact is more in the older age group and retired populations. Also public health facilities already groaning under the heavy burden of communicable diseases care' are unable to meet the need for people that require care for CVDs e.g. MI, stroke and ischæmic heart diseases (IHD). If countries in SSA are to make available half of the resources budgeted for health care for CVDs in high income countries, their economies will probably crumble.

The aim of this survey was to determine the prevalence of CVD risk factors among women attending a religious outreach program in Ekiti State, Nigeria.

\section{Methodology}

The study was conducted in Ado - Ekiti, a state capital city in the south western zone of Nigeria. Ado Ekiti, is an urban settlement with a current estimated population of about 366,280 [8] Ekiti State University and the Ekiti State University Teaching Hospital are located in the town. Other tertiary institutions include the Afe Babalola University, the Federal polytechnic and the Crown Polytechnic. The major occupations of the residents are trading, semi-skilled work and public service. The study design was a descriptive crosssectional survey. The participants recruited forthe study were adult women that were participating in a religious outreach program organized by a prominent Christian denomination in Nigeria where free medical services were provided. The minimal sample size for the study was estimated at 386 using the modified Leslie Fisher's formula for single proportions. [89] However, 426 women were randomly recruited to participate in the study. Interviewer administered semi - structured questionnaires were used to collect information on respondents socio-demographic characteristics, past medical history, nutritional status (using dietary recall), and behaviors related to lifestyle. A general physical examination was done and anthropometric measurements taken from each respondent. The examinations collected data on, blood pressure, weight and height. Blood specimen ( $5 \mathrm{ml}$ whole blood) was collected from each respondent on which laboratory tests were performed. Data obtained from the laboratory tests included random blood sugar (RBS) and serum cholesterol levels. Data from the clinical examinations and laboratory tests were then used to categorize respondents as hypertensive or not, diabetic or not, obese or not and hyperlipidemic or not. Hypertension was assessed among respondents using a benchmark value of 140/90 $\mathrm{mmHg}$. Respondents were considered probably diabetic if they had a random blood glucose level of above $11.1 \mathrm{mmol} / \mathrm{L}$, while respondents with a BMI of $30 \mathrm{~kg} / \mathrm{m}^{2}$ and above were categorized as obese. Respondents with serum lipid (LDL) values as stated below were considered to be abnormal values.

Total Cholesterol: above $200 \mathrm{mg} / \mathrm{dL}(5.18 \mathrm{mmol} / \mathrm{L})$

HDL Cholesterol: Less than40mg /dL (1.0 mmol/L ) for men and less than $50 \mathrm{mg} / \mathrm{dL}(1.3 \mathrm{mmol} / \mathrm{L})$ for women

Fasting Triglycerides: above $150 \mathrm{mg} / \mathrm{dL}(1.70 \mathrm{mmol} / \mathrm{L})$

LDL Cholesterol: above $100 \mathrm{mg} / \mathrm{dL}$ (2.59 mmol/L)

Smokers were identified by eliciting history of active cigarette smoking.

Respondents were thereafter assessed as being at risk or not at risk of CVD disease based on the computation of a composite score derived from the allocation of a value of 0 or 1 to outcome measures of blood pressure, body mass index, random blood glucose, serum lipid levels and tobacco use (risk factors). A value of between 0 and 1 was attached to the presence or absence of a risk factor. For example respondents that were hypertensive, diabetic, obese, having high serum lipids or smokers were ascribed a value of 1 (such that any respondent with all the risks had a maximum score of 5 while a respondent without any of the risks had a score of 0 ). Respondents with score of 3 and above were then labeled to be at an increased risk of developing cardiovascular disease. Data was analyzed using SPSS version 20 and results presented as prose, tables and charts. Association between variables of interest was explored using the chi square tests and level of significance was set at $p$ values $<0.5$. Informed consent was obtained from participants verbally and in written form before interview, physical examination and collection of blood specimens. An ethical approval was obtained from the ethical and research committee of EKSUTH before commencement of the study. Participation in the study was entirely voluntary and confidentiality was observed.

\section{Results}

A total of 426 respondents participated in the survey. 
Table 1. Socio-Demographic Characteristic of Respondents.

\begin{tabular}{|c|c|c|}
\hline Socio- Demographic Characteristics & Frequency & Percentage $\%$ \\
\hline \multicolumn{3}{|l|}{ Age (years) } \\
\hline $20-29$ & 12 & 2.8 \\
\hline $30-39$ & 39 & 9.2 \\
\hline $40-49$ & 123 & 28.9 \\
\hline $50-59$ & 153 & 35.9 \\
\hline 60 and above & 99 & 23.2 \\
\hline \multicolumn{3}{|l|}{ Educational Status } \\
\hline No formal Education & 78 & 18.3 \\
\hline Primary & 51 & 12.0 \\
\hline Secondary & 105 & 24.6 \\
\hline Tertiary & 192 & 45.1 \\
\hline \multicolumn{3}{|l|}{ Occupational Status } \\
\hline Not employed & 15 & 3.5 \\
\hline Unskilled (traders / laborers ) & 213 & 50.0 \\
\hline Skilled (artisans / professionals) & 198 & 46.5 \\
\hline \multicolumn{3}{|l|}{ Family size } \\
\hline None & 132 & 31.0 \\
\hline $1-4$ & 138 & 32.4 \\
\hline 5 and above & 156 & 36.6 \\
\hline \multicolumn{3}{|l|}{ Average Monthly family Income } \\
\hline None & 180 & 42.3 \\
\hline$<20,000$ & 69 & 16.2 \\
\hline $20,000-200,000$ & 171 & 40.1 \\
\hline$>200,000$ & 6 & 1.4 \\
\hline \multicolumn{3}{|l|}{ Marital Status } \\
\hline Single & 3 & 0.7 \\
\hline Married & 414 & 97.2 \\
\hline Widow & 9 & 2.1 \\
\hline \multicolumn{3}{|l|}{ Family type } \\
\hline Monogamy & 339 & 79.6 \\
\hline Polygamy & 87 & 20.4 \\
\hline Total & 426 & 100 \\
\hline
\end{tabular}

Majority of respondents, $64.8 \%$ were between $40-59$ years with amean age of $51.7 \pm 11.9$ years. A high proportion $(81.7 \%)$ of respondents had formal education. Only $57.7 \%$ of participants gave information on their monthly income and of these, majority claimed they earned an average of between $\mathrm{N}$ $20,000.00$ and $N 200,000.00$. The mean income of the respondents was $\mathrm{N} \mathrm{81,622}$ (\$400). Nearly all (97.2\%) the respondents were married with about $79.6 \%$ in monogamous relationships. Most of the respondents (95\%) claimed to be employed with about $50 \%$ of them being engaged in semiskilled work e.g. trading, tailoring, hair dressing.

A little over half $(51.4 \%)$ of the respondents had a substance use habit. The most common substances regularly consumed by respondents were "bitter kola" (31.7\%) and"kolanut" (9.9\%). About 5\% of respondents either smoked or took substances containing nicotine e.g. "snuff ". Nearly a quarter, $23.2 \%$ of respondents claimed that they have ever used used herbal (traditional) medicine $(23.2 \%)$ to take care of health issues as the need arises. Also, about 9.2\% of respondents claimed that they occasionally consumed alcoholic drinks. Majority (90.1\%) of respondents had access to at least to some form of domestic water supply and the most common source was the sanitary well $(69.0 \%)$.

Assessment of nutritional intake was done by collecting information on the weekly consumption pattern and a 3 day total and protein dietary recall of respondents. Based on this only $52 \%$ of the respondents were on a diet that could be considered to be balanced (Figure 1).

\section{Medical History}

Respondents were categorized as having a good or poor medical history based on information collected on previous admissions for medical or surgical conditions and having a chronic medical condition for which they were receiving treatment - and almost half (49.5\%) of the respondents were found to have a poor medical history. About $46.5 \%$ of respondents had a history of at least one episode of hospitalization. However, the commonest reason for hospitalization was for pregnancy related issues $(15.5 \%)$ and malaria (14.1\%). Also, about $40.8 \%$ of respondents reported having health conditions for which they currently are in need of intervention - these conditions include malaria (15.5\%), peptic ulcer disease $(2.8 \%)$, hypertension $(1.4 \%)$, ophthalmic conditions $(4.9 \%)$ and non-specified medical conditions $(15.5 \%)$.

Table 2. Cardiovascular risk assessmentand distribution of risk factors among respondents.

\begin{tabular}{llc}
\hline CVD Risk factors & Frequency & \% \\
\hline BMI & 12 & \\
Under weight & 153 & 3.8 \\
Normal & 162 & 38.9 \\
Mild obesity & 75 & 17.6 \\
Moderate Obesity & 24 & 5.6 \\
Severe Obesity & & \\
BP & 315 & 73.9 \\
Normal & 51 & 12.0 \\
High normal (pre-hypertension) & 27 & 6.3 \\
Mild hypertension & 30 & 7.0 \\
Moderate hypertension & 3 & 0.7 \\
Severe hypertension & & \\
Random Blood Glucose & 117 & 27.5 \\
High & 309 & 72.5 \\
Normal & & \\
Serum Lipid (LDL) & 156 & 36.6 \\
High & 270 & 63.4 \\
Normal/ Low & & \\
Smoking Status & 9.0 & 2.1 \\
Yes & 417 & 97.9 \\
No & & \\
CVD Risk & 300 & 100 \\
Low Risk & 126 & \\
High Risk & 426 & \\
Total & & \\
\hline
\end{tabular}

Risk for future development of CVD was assessed by studying certain risk factors i.e. blood pressure (BP), BMI, random blood sugar (RBS), blood lipid (BL) and smoking. While majority $(73.9 \%)$ of the respondents had a normal blood pressure (BP) $-12.0 \%$ and $14 \%$ were either prehypertensive or hypertensive. Majority of the respondents were either pre-obese $(38 \%)$ or moderately obese $(17.6 \%)$ only $35.9 \%$ of the respondents had a normal BMI. About $27.5 \%$ of the respondents had high random blood sugar levels. A high proportion of respondents $(63.4 \%)$ had high lipid (LDL) levels. Prevalence of smoking among the respondents was low $(2.1 \%)$. 
Respondents were then assessed as having a future risk of developing cardiovascular disease based on certain variables i.e. outcome of physical examination (BP and BMI), laboratory investigations (random blood glucose and LDL levels) and smoking. Based on these evaluations, about $29.6 \%$ of the respondents were found to be at risk of future CVD and will require one or more forms of intervention (Table 2).

Table 3. Socio-demographic characteristics as related to CVDrisk.

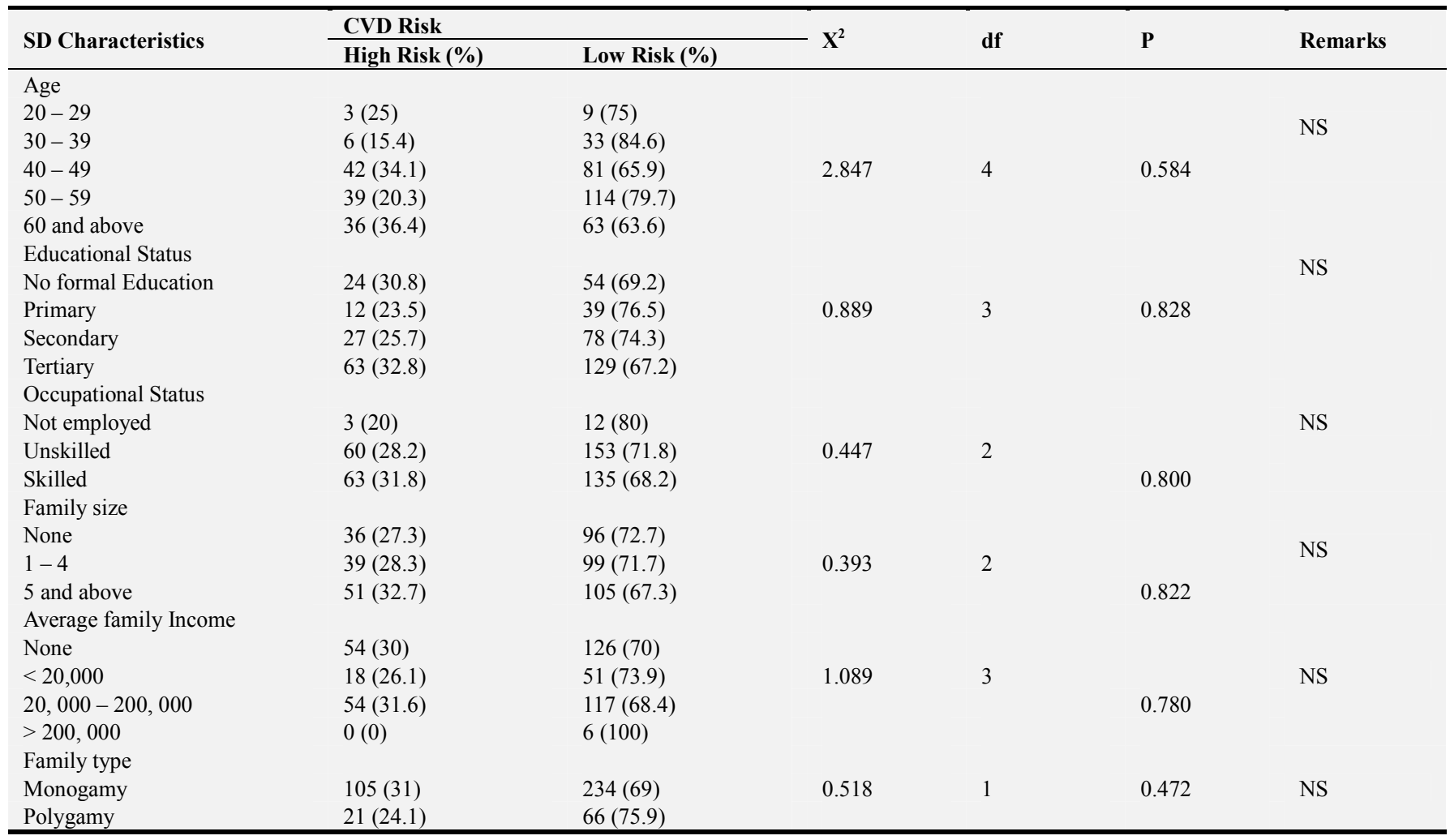

The association between socio-demographic characteristics and cardiovascular disease risk of respondents was explored. It was found that women that were younger, educated and employed had a lower CVD risk when compared to those that were older, less educated and unemployed. Though the relationships were not statistically significant $(p>0.05)$

\section{Discussion}

In this study we set out to assess the risk of future development of CVD and also determine the prevalence of risk factors among women attending a religious outreach program in Ado-Ekiti, Ekiti State, Nigeria.

Age

The respondents had a mean age of $51.7 \pm 11.9$ years - this indicates a group of people who are at risk of future development of a CVD. Even though Nigeria has a relatively young population, the large number of people (182.2 million) associated with high fertility (5.7), growth rate (2.8), improving survival into adulthood and exposure to risk factors (e.g urbanization and westernization ) implies that a large proportion are exposed to the riskof developing CVD in the nearer future. [8-9]

A larger proportion of the young people in Nigeria, are now surviving into adulthood due to the success of child survival programs. Recent projections reveal that about 15 -
$64 \%$ of the population are found between the age of 15-64 years. [9] with 40 to50 years being the critical age at which the incidence of CVD increase world- wide. [10] In consonance with what is happening in other countries ofSSA, the burden of CVD in Nigeria is higher among relatively younger age group, when compared to high income countries that have it among the elderly, with its attendant negative effect on productivity and development of a country already burdened with high prevalence of infectious diseases. [2].

Age is a factor in the development of CVD as the degenerative changes that occur due to exposure to environmental risks accumulate with advancing age. While age in of itself is important, the relevant exposure is the lifecourse accumulation of risk factors that is afforded by longevity. [6] Projections from the Global burden of disease reports show that a large proportion of the future victims of CVD in SSA will be middle aged people. [3] In sub-Sahara Africa, similar to other global trends, changing demographic profile due to greater survival into adulthood as a result of reduction in the burden of communicable diseases in childhood is central to the increasing prevalence of cardiovascular disease in the region. [7] While changes have occurred in the environmental and behavioral determinants of CVD such as tobacco use, increasing fat and calorie consumption and decreasing exercise, longer periods of exposure to the these determinants because of longer life 
expectancy have increased the rates of chronic disease. [6] In developed countries, screening and interventions programs for prevention of cardiovascular disease usually start at the age of 20 and 50 years respectively, to achieve optimal outcomes. [11].

Currently though the most common causes of morbidity and mortality among women in sub-Saharan Africa is still from infections and pregnancy related events, noncommunicable diseases like hypertension, diabetes mellitus (DM), cancer and cardiovascular disease are increasingly becoming prevalent. [3, 4] Current statistics reveal that among older women (60 years and above), noncommunicable diseases, particularly CVD and cancers, are the most important causes of death, regardless of the level of economic development of the country in which they live. [3, 4] Cardiovascular diseases account for $46 \%$ of older women's deaths globally - while a further $14 \%$ and $9 \%$ are caused by cancers and chronic respiratory conditions respectively. Many of the health problems faced by women in older age are the result of exposure to risk factors in adolescence and adulthood, such as smoking, sedentary lifestyles and unhealthy diets. [12]

In this study, about $29.6 \%$ of the respondents were found to be at an increased risk of developing cardiovascular disease. Many studies conducted in Nigeria and other SSA countries reveal a similar trend. For example a study in Enugu, Nigeria revealed a high prevalence of CVD among patients that were admitted in a tertiary health institution, concluding that CVDs were a major cause of morbidity and mortality in the environment. [13] Similar trends were observed from other locations in Nigeria. [14].

The modifiable CVD risk factors investigated in this study were blood pressure, blood sugar levels, body mass index (BMI), blood lipid levels and smoking. Among the respondents about $26.1 \%, 26.1 \%, 61.3 \%$ and $36.6 \%$ had high blood pressure, high random blood sugar (high RBS), obese and hyperlipidemic respectively - only about $2.1 \%$ of them were smokers. The increasing modifiable CVD risk factor prevalence found in this study is consistent with current findings in Nigeria. [15] Also, the result of a multicenter study and other cross sectional surveys in specific locations in SSA indicate that hypertension is probably the most important factor driving CVDs resulting in the different trend of stroke being the most prevalent CVD in Africa, as opposed to myocardial infarction in developed high income countries. $[3,5,6]$ Cigarette smoking and use of tobacco products is one of the major modifiable CVD risk factor prevalent especially in high income countries. [16 57] It is a global public health challenge and is a major contributor to the pattern of CVD seen in these countries i.e. ischeamic heart disease (IHD) and myocardial infarction (MI). ${ }^{17}$ The prevalence of smoking in this study was found to be low at about $2.1 \%$. In Nigeria the use of tobacco is still relatively low compared to other countries in sub-Sahara Africa and regions of the world at $16.4 \%$ and $1.2 \%$ for males and females respectively. [17] However current data suggest an increasing prevalence of smoking among younger people due to the effect of western culture and urbanization.

In this study, it was found that women that were younger, educated and employed had a lower CVD risk when compared to respondents that were older, less educated and unemployed.-though the relationships were not statistically significant $(\mathrm{p}>0.05)$. Age is an important non-modifiable risk factor for cardiovascular disease. As people get older, the exposure to environmental and social CVD risk factors increase and the accumulation of these results in a higher prevalence of CVD. [10] However being educated and employed may be a mitigating factor as these are known positive social determinants for health.

\section{Conclusion}

The Study concluded that there were significant risk factors among women in Ekiti State. Obesity and high serum cholesterol were predominantly noticed risk factors identified among others. Health education becomes a crucial aspect of intervention to control for the prevalence of these risk factors coupled with regular comprehensive medical check up.

\section{References}

[1] Moshe Naghavi, Mohammed L Forouzanfar. Burden of noncommunicable diseases in sub-Saharan Africa in 1990 and 2010: Global burden of diseases, injuries and Risk factors study 2010. The Lancet, 2013, 381, Special issue S95.

[2] Dalal S. Beunza JJ, Volmink J, Adebamowo C, Bajunirwe F, Njeleka M, Mozaffarian D, Fawzi W, Willet W, Adami H and Holmes MD. Non communicable diseases in sub-Saharan Africa: what we know now. Int J Epidemiology 2011:40 (4): 885-901.

[3] Lopez AD, Mathers CD, Ezzati M, Jamison DT, Murray CJ. Global and regional burden of disease and risk factors, 2001: systematic analysis of population health data. Lancet 2006; 367:1747-57.

[4] Yach D, Hawkes C, Gould C, Hoffman K. the Global burden of Chronic diseases: overcoming Impediments to prevention and control. JAMA. 2004n, 291 (21): 2616 - 2622.

[5] Sampson Uk, Amuyyunzu - Nyamago GA, Mensah GA. health promotion and cardiovascular disease prevention in sub-Saharan Africa.. Prog Cardiovascular disease. 2013: 56(3): 344-55.

[6] Tourlouki E, Matalas AL, Panagiotakos DB. dietary habits and and cardiovascular disease risk factors in middle aged and elderly populations: a review of evidence. Clin Interv Aging. 2009; 4:319-30.

[7] Fuster V, Kelly BB (eds). Promoting cardiovascular health in the developing world: A critical challenge to achieve global health. Washington, DC: The National Academies Press, 2010.

[8] Fishers A., Lang J. Sample and sample size determination in research designs, 1963. The Population Council, New York.

[9] National Population Commission Nigeria and ICF International. 2014. And Nigerian Demographic and Health Survey 2013. 
[10] Wald NJ, Simmonds M, Morris JK (2011) Screening for Future Cardiovascular Disease Using Age Alone Compared with Multiple Risk Factors and Age. PLoS ONE 6 (5): e18742 doi 10.1371/journal.pone 0018742 .

[11] American Heart Association, 2016. heart health Screenings. http: //www. heart.org/HEARTORG/Conditions/HeartHeaalth-screening. Accessed $12^{\text {th }}$ Dec 2016.

[12] World Health Organisation. Womens Health. Fact Sheet 334. http://www.who.int/mediacenter/factsheets/fs334/en/.

[13] Oguanobi NI, Ejim EC, Onwubere BJ, Ike SO, Anisiuba BC, Ikeh VO, Aneke EO, Pattern of Cadiovascular Disease amongst Medical admissions in a regional Teaching Hospital in South-eastern Nigeria. Nig J Cardiol 2013;10:77-80.

[14] Okesina AB, Oparinde DP, Akindoyin KA, Prevalence of some of the risk factors of Coronary Heart Disease in a Rural Nigerian Population. East Afr Med J. 1999;76:212-216.
[15] Kadiri S, Salako B, cardiovascular risk factors in middle age Nigerians. East African Medical Journal. 1997, 74 (5); 303 306.

[16] Havranek EP, Mujahid MS, Barr DA, Blair IV, Cohen MS, Cruz-Florez S, Davey- Smith G, et al. Social Determinants and Risk Outcomes for Cardiovascular Disease. Circulation. 2015; $\quad 132: \quad 873 \quad-\quad 898$ htts://doi.org/10.1161/CIR.00000000000000228.

[17] Sani MU, Adamu B, Mijinyawa MS, Abdu A, Kararye KM, Maiyaki MB, et al. Ischaemic Heart Disease in Aminu Kano Teaching Hospital, Kano Nigeria:A 5 year review. Nig J Med 2006; 15: 128-31. 